\title{
Osteogenesis Imperfecta in Pregnancy. Case Report
}

\author{
Ticiana Goyanna Lyra ${ }^{1}$, Vanessa Alves Fernandes Pinto ${ }^{2}$, Fábio André Barbosa Ivo ${ }^{3}$, Jedson dos Santos \\ Nascimento, TSA 4
}

Summary: Lyra TG, Pinto VAF, Ivo FAB, Nascimento JS - Osteogenesis Imperfecta in Pregnancy. Case Report.

Background and objectives: Osteogenesis Imperfecta is a rare condition, especially in obstetric patients. It has an estimated prevalence of $1 / 10,000$ in the general population, and $1 / 25,000$ to 30,000 in obstetric patients. The objective of this report was to present a rare case of a pregnant woman with Osteogenesis Imperfecta undergoing cesarean section.

Case report: This is a 23 years old gravida on the $38^{\text {th }}$ week, admitted to the maternity ward with loss of amniotic fluid and contractions for four hours, associated with the absence of fetal movements for four days. The patient had a mild form of Osteogenesis Imperfecta without other comorbidities. She was not taking any medication, and she did not have prenatal follow-up. The patient underwent spinal anesthesia in the $L_{3}$ - $L_{4}$ space, median approach, with a single puncture with a 27G Quincke needle and the administration of hyperbaric $0.5 \%$ bupivacaine (10 mg) and morphine $(60 \mu \mathrm{g})$. She was discharged in the second postoperative day without complaints.

Conclusions: Fertility is preserved, especially in those patients with type I of the disease, and pregnancy can be carried to term. Delivery is usually surgical due to pelvic deformities in the gravida, cephalopelvic disproportion, and increased incidence of abnormal fetal presentation. The importance of the anesthesiologist in the surgical team relies on the perioperative management and the choice of the most appropriate anesthetic technique for each patient.

Keywords: ANESTHETIC TECHNIQUES, Regional: subarachnoid; DISEASES, Genetic: osteogenesis imperfecta; SURGERY, Obstetric: cesarean section.

[Rev Bras Anestesiol 2010;60(3): 321-324] CElsevier Editora Ltda.

\section{INTRODUCTION}

Osteogenesis Imperfecta $(\mathrm{OI})$ is a rare condition, especially in obstetric patients. It has an incidence of $1 / 10,000$ in the general population, and $1 / 25,000$ to 30,000 in obstetric patients ${ }^{1,2}$. It results from mutations in collagen genes involved in endochondral ossification, with consequent bone fragility and multiple spontaneous fractures or after mild traumas ${ }^{3}$. It has several clinical presentations, according to the severity of the involvement. Type I is the most common with mild involvement without major deformities, and normal stature. Type II is the most severe affecting neonates, and it is usually incompatible with life. In type III patients have short stature, triangular fascies, and bone deformities. Type V, OI with hypertrophic calluses and calcification of the interosseous membrane of the forearm was recently described in the literature ${ }^{3}$.

Received from the Maternidade de Referência Professor José Maria de Magalhães Netto, CET/SBA of the Santa Casa de Misericórdia da Bahia, Salvador, BA

1. ME2 of the CET/SBA of the Santa Casa de Misericórdia da Bahia

2. Anesthesiologist of the Maternidade de Referência Professor José Maria de Magalhães

Netto

3. ME3 of the CET/SBA Obras Sociais Irmã Dulce

4. Professor, PhD in Anesthesiology; Responsible for the CET/SBA Santa Casa de Misericórdia da Bahia

Submitted on June 24, 2009

Approved on February 3, 2010

Correspondence to:

Dra. Ticiana Goyanna Lyra

Serviço de Anestesia - Hospital Santa Izabel

Praça Almeida Couto, $s / n^{\circ}-$ Nazaré

40050-405 - Salvador, BA, Brasil

E-mail: ticig@yahoo.com.br
The importance of this subject in the literature lies in the rarity of the case and scarcity of studies published in anesthesiology journals. The objective of this report was to present the case of a gravida at term with Osteogenesis Imperfecta who underwent cesarean section under subarachnoid block.

\section{CASE REPORT}

This is a 23 years old gravida, gesta 2 to 1 , gestational age 38 weeks with history of a cesarean section one year ago, with loss of amniotic fluid and contractions for 4 hours and absence of fetal movements for 4 days. On physical exam, fetal heart beats were not audible, and the patient was in labor. The patient had a mild type of OI without associated comorbidities; she was not taking any medication and she did not have any prenatal follow-up. The patient had mild deformities in the upper and lower limbs and lumbar scoliosis. She had a history of surgeries for fractures of the femur and hip. The patient was hemodynamically stable, being admitted for a cesarean section. Laboratorial exams (CBC, renal function, blood glucose levels, and clotting) did not show abnormalities, and negative serologies (HIV, VDRL, and HBsAg).

The patient was monitored with continuous cardiograph, pulse oximeter, and non-invasive blood pressure. An 18G catheter was used for venipuncture, and oxygen, 3 L.min ${ }^{-1}$, was administered via a nasal cannula. A 27F Quincke needle was used for the median puncture in the $\mathrm{L}_{3}-\mathrm{L}_{4}$ space, and hyperbaric $0.5 \%$ bupivacaine $(10 \mathrm{mg})$ and morphine $(60 \mu \mathrm{g})$ were administered. Dexa- 
methasone (4 mg) and cefalotin (2 g) were administered before the procedure, oxytocin (5 IU) was administered intraoperatively, and dypirone (2 g), ketoprofen (100 mg), and ondansetron (4 mg) were administered at the end of the procedure. Ringer's lactate $1,000 \mathrm{~mL}$, and NS $500 \mathrm{~mL}$, were administered for hydration. The patient remained hemodynamically stable throughout the intra- and postoperative periods, and she was discharged on the second postoperative day without any complaints.

\section{DISCUSSION}

Other clinical characteristics such as bluish sclera, hyperhidrosis, conduction deafness, hyperthermia, odontological deformities, platelet dysfunction, congenital cardiopathy, valvular cardiopathy, cor pulmonale, and joint and dermatological disorder can be associated with $\mathrm{OI}^{4}$.

Fertility is preserved especially in patients with OI type I, and pregnancy can be carried to term. The incidence of fractures is not increased during pregnancy. Due to pelvic deformities of the gravida, increased incidence of cephalopelvic disproportion, and abnormalities in fetal presentation, delivery is usually surgical 4,5 . Treatment with biphosphonates before pregnancy is controversial due to the interference with maternal calcium metabolism and changes in fetal bone modelling. Case reports of prolonged biphosphonate treatment before conception have not observed maternal or fetal adverse reactions ${ }^{6}$.

The fetal diagnosis can be done through chorionic villus sampling and imaging exams of the fetus. If fetal $\mathrm{OI}$ is confirmed pre-natally, especially the lethal type, cesarean section is not indicated because, besides increasing maternal morbidity, it does not improve fetal prognosis. Cesarean sections do not decrease the number of fetal fractures in newborns with nonlethal types of $\mathrm{OI}^{5}$.

The anesthesiologists must take steps to prevent further damage to patients, such as placing pads on the surgical table, avoid hyperinflation of the sphygmomanometer, and use of gentle movements during laryngoscopy. Invasive blood pressure should be considered in patients with severe OI. Abnormalities of the spine and neural compression secondary to multiple fractures can hinder neuroaxis block ${ }^{1}$. During pregnancy, reduction in physiological residual capacity can be added to the reduction in vital capacity secondary to restrictive pneumopathy due to multiple vertebral and rib fractures.

In general anesthesia, topical anesthesia of the oropharynx should be used before laryngoscopy with awaken patient to evaluate the difficulty of the intubation. Assisted intubation by fiberoptic bronchoscopy and the use of the laryngeal mask should be considered ${ }^{4}$. Anatomical changes such as short neck, temporal and occipital projections, and prominent mandible could lead to difficult intubation. Teeth, mandibular, and cervical fractures, and mucous hemorrhage can result from multiple attempts to intubate those patients ${ }^{7}$. Inhalational agents and succinylcholine can trigger malignant hyperthermia, even in those patients who are not genetically susceptible $^{8}$.

In spinal anesthesia, besides the technical difficulties, spinal deformities make the level of the blockade unpredictable. Continuous spinal anesthesia with epidural or subarachnoid catheter is a safer option.

Excessive bleeding and even disseminated intravascular coagulation can be seen in patients with $\mathrm{OI}$ undergoing surgical procedures. Capillary fragility, reduction in the levels of factor VIII and platelet activity secondary to deficient collagen binding can be present. This collagen deficiency leads to friable tissues and inadequate vasoconstriction in response to bleeding ${ }^{7}$. Pregnant women are much more prone to uterine atony postpartum.

This report described a rare case of $\mathrm{OI}$ in a pregnant patient who underwent urgent cesarean section. The importance of the anesthesiologist in the team relies on the perioperative management and the choice of the most appropriate anesthetic technique for each patient. 